\title{
La búsqueda de sensaciones y su relación con la vulnerabilidad a la adicción y al estrés
}

\author{
Roser Nadal Alemany* \\ *Profesora Titular de Universidad. Universidad Autónoma de Barcelona. Instituto de Neurociencias y Unidad de Psicobiología (Facultad de Psicología) \\ Enviar correspondencia a: \\ Roser Nadal Alemany. Instituto de Neurociencias y Unidad de Psicobiología (Facultad de Psicología). Universidad Autónoma de Barcelona. 08193 Bellaterra. \\ Barcelona. Correo e.: roser.nadal@uab.es
}

\section{RESUMEN}

Existe una importante vulnerabilidad individual a la adicción que puede estar relacionada con factores de personalidad. Uno de los rasgos de personalidad más estudiado en relación a la susceptibilidad a la adicción ha sido el de búsqueda de sensaciones o de novedades. En esta revisión se analizan principalmente datos de investigación básica sobre la relación entre adicción y búsqueda de sensaciones. En modelos animales este rasgo ha sido valorado principalmente mediante la actividad motora en un ambiente nuevo, estableciéndose dos tipologías de ratas, las denominadas LR (low-responders) y las HR (high-responders) que muestran respectivamente niveles bajos y altos de actividad en dicho ambiente nuevo. Los animales HR son más sensibles a los efectos estimulantes motores de diversas drogas y muestran mayor sensibilización conductual ante la administración repetida de la droga respecto a los LR. Además, los sujetos HR adquieren inicialmente más fácilmente la conducta de autoadministración operante de diversas drogas de abuso. La reactividad del eje hipotálamo-pituitario-adrenal ante ciertas situaciones de estrés también es mayor en los animales HR que en los LR A nivel del sistema nervioso central, los sujetos HR también presentan una mayor actividad del eje hipotalámico-pituitarioadrenal. Respecto al sistema dopaminérgico, estudios de microdiálisis muestran que la cocaína ejerce un mayor efecto neuroquímico (mayor incremento en los niveles de dopamina) en los animales HR que en los LR. Estos datos en animales a grandes rasgos se replican también en humanos, aunque existen también resultados contradictorios tanto en animales como en humanos, indicando que el tema es complejo y aún no está resuelto.

Palabras clave: Personalidad, diferencias individuales, estrés, vulnerabilidad, búsqueda de sensaciones.

\section{ABSTRACT}

There is considerable variation in individual vulnerability to addiction that may be related to personality factors. One of the most widely studied personality traits in relation to susceptibility to addiction has been novelty or sensation-seeking. In the present review we mainly analyze basic research data on the relationship between addiction and novelty-seeking. In animal models, this trait has been assessed mainly by means of motor activity in an inescapable novel environment. Thus, two typologies of rats have been established, the so-called LR (lowresponders) and the HR (high-responders), which respectively show low and high levels of activity in a novel environment. $\mathrm{HR}$ animals are more sensitive to the motor-stimulating effects of various drugs, and show greater behavioural sensitization with repeated drug administration, than LR animals. Moreover, $H R$ subjects initially acquire operant self-administration behaviour more easily than LR subjects. Hypothalamicpituitary-adrenal axis reactivity in certain stressful situations is also greater in HR than in LR subjects. At the level of the central nervous system, HR subjects also show greater activity of the hypothalamic-pituitary-adrenal axis. With regard to dopaminergic systems, microdialysis studies show that cocaine has a greater neurochemical effect (more increase in dopamine levels) in HR than in LR animals. Human data are generally in agreement with those from the animal literature, though there are also contradictory results in both humans and animals, suggesting that this topic is complex and still far from being fully understood.

Key words: Personality, individual differences, stress, vulnerability, sensation-seeking. 


\section{INTRODUCCIÓN}

L a existencia de una vulnerabilidad individual a la adicción podría estar relacionada con factores de personalidad. Uno de los más estudiados en relación a la susceptibilidad a la adicción ha sido el de novelty-seeking, también denominado sensationseeking, o la búsqueda de novedades, sensaciones o experiencias nuevas, variadas y en ocasiones arriesgadas o peligrosas. El primer autor que propuso el término fue Zuckerman (ver revisión en Zuckerman, 1986) y posteriormente fue desarrollado por otros autores como Cloninger (1994). Nos referiremos en esta revisión a la relación de este rasgo con la vulnerabilidad a la adicción y la reactividad al estrés, tanto en modelos animales como en humanos (ver Tabla 1 para un resumen de los principales resultados).

\section{MODELOS ANIMALES}

La búsqueda de novedad o de sensaciones se ha intentado modelar en animales mediante dos aproximaciones metodológicas distintas que no son necesariamente paralelas. Una de ellas es el estudio de la reactividad motora en un ambiente nuevo en el que el animal no tenga escapatoria durante un determinado período de tiempo (Dellu, Piazza, Mayo, Le Moal y Simon, 1996b; Piazza, Deminière, Le Moal y Simon, 1989). Otra estrategia es la de estudiar la preferencia del animal por situaciones u objetos nuevos versus otros ya conocidos (Bardo, Donohew y Harrington, 1996). Es difícil establecer cuál de las dos aproximaciones es más correcta. Además, los ambientes nuevos a los que son expuestos los animales pueden ser muy distintos en función del equipo de investigación que realiza el estudio e incluso los tiempos de exposición al ambiente nuevo varían desde cinco minutos a dos horas, con lo que probablemente se estén midiendo aspectos distintos del comportamiento del animal (Marinelli, 2005).

Respecto al tipo de población estudiada, se han utilizado habitualmente ratas outbred no seleccionadas genéticamente, aunque existen también algunas cepas de animales outbred seleccionados genéticamente (por ejemplo, las ratas APO-SUS y APO-NonSUS, seleccionadas por su sensibilidad a la apomorfina, Ellenbroek, van der Kam, van der Elst y Cools, 2005; o las ratas RHA y RLA, seleccionadas por su ejecución en una tarea de evitación activa de dos sentidos, Steimer y Driscoll, 2003) y algunas cepas consanguíneas (por ejemplo, los ratones DBA/2 y C57BL/6, Cabib, Orsini, Le Moal y Piazza, 2000) que difieren en su grado de búsqueda de sensaciones.

Con referencia a la edad de lo sujetos utilizados, cabe mencionar que la adolescencia es una etapa ontogenética donde el rasgo de búsqueda de sensaciones es más marcado, tanto en animales, como en humanos (Laviola, Macri, Morley-Fletcher y Adriani, 2003). Incluso el rasgo de búsqueda de sensaciones

Tabla 1. Resumen de los principales resultados respecto a la relación del rasgo de búsqueda de sensaciones en animales con la susceptibilidad al estrés y a la adicción.

\begin{tabular}{|l|l|}
\hline \multirow{2}{*}{ Métodos de medida } & $\begin{array}{l}\text { Actividad motora en ambientes nuevos (animales con mayor exploración o HR, y animales con menor explo- } \\
\text { ración o LR). } \\
\text { Preferencia por ambientes u objetos nuevos. }\end{array}$ \\
\hline Respuesta a las drogas & $\begin{array}{l}\text { Los animales HR se autoadministran inicialmente más cantidad de droga. } \\
\text { Los HR tienen más sensibilidad a los efectos estimulantes motores de las drogas. }\end{array}$ \\
\hline Respuesta al estrés & $\begin{array}{l}\text { Los HR muestran mayor liberación de corticosterona y de ACTH tras la exposición a ciertas situaciones de } \\
\text { estrés. }\end{array}$ \\
\hline Substrato neurobiológico & $\begin{array}{l}\text { Los HR presentan una mayor respuesta neuroquímica a nivel de la dopamina en respuesta a los psicoestimu- } \\
\text { lantes que los LR. } \\
\text { Los HR presentan un mayor funcionamiento del eje hipotalámico-pituitario-adrenal a nivel del sistema nervio- } \\
\text { so central que los LR. }\end{array}$ \\
\hline
\end{tabular}


durante la adolescencia predice en humanos un mayor abuso de ciertas drogas, como el alcohol (Cloninger, Sigvardsson y Bohman, 1988). Sin embargo, la mayoría de los estudios sobre la relación de este rasgo con la susceptibilidad a la adicción y al estrés se han centrado en animales adultos.

El laboratorio que inició los estudios sobre las diferencias individuales en reactividad motora y su relación con la respuesta a las drogas y con la susceptibilidad a la adicción y al estrés fue el de Le Moal y Piazza a finales de los 80 en Burdeos (ver revisiones en Dellu, Mayo, Piazza, Le Moal y Simon, 1993; Dellu et al., 1996b). Posteriormente otros laboratorios han extendido sus trabajos, encontrándose algunos datos contradictorios.

\section{Modelos de búsqueda de sensaciones en situación inescapable}

Búsqueda de sensaciones y su relación con la respuesta a las drogas

El equipo de Le Moal y Piazza partía de una población de ratas Sprague-Dawley outbred no seleccionadas genéticamente y caracterizaban su actividad motora en un ambiente nuevo en el que el animal no tenía escapatoria (un corredor circular) durante un período de tiempo habitualmente entre 1 y 2 h. Posteriormente dividían los animales según la mediana en dos grupos, los denominados LR (low-responders) y los HR (high-responders), según mostraran una baja o alta actividad motora en dicho ambiente. Este rasgo de reactividad en ambientes nuevos se consideró análogo de la dimensión humana de personalidad de búsqueda de sensaciones (ver revisiones de Dellu et al., 1996b; Kabbaj, 2004, 2006).

En un influyente artículo en Science, el laboratorio de Burdeos demostró que esta actividad motora en ambientes nuevos estaba relacionada con la actividad motora inducida por la anfetamina y con la autoadministracíón intravenosa de la droga en una situación operante en un paradigma en el que se daba acceso a la droga en cinco sesiones en un programa de reforzamiento contínuo (Piazza et al., 1989). Por otra parte, es muy interesante mencionar que el tratamiento previo de administración repetida de anfetamina diluía las diferencias individuales en autoadministración de anfetamina presentadas por ambos grupos. Esta mayor susceptibilidad individual en la adquisición de la autoadministración operante de anfetamina de los animales HR se replicó en otros estudios posteriores del mismo equipo (Piazza, Deminière, Maccari, Mormède, Le Moal y Simon, 1990, Piazza, Maccari, Deminière, Le Moal, Mormède y Simon, 1991) y se generalizó para la cocaína (Piazza, Deroche-Gamo- nent, Rougé-Pont y Le Moal, 2000). Otros laboratorios han replicado también estos datos con drogas como la anfetamina (Cain, Denehy y Bardo, 2007; Pierre y Vezina, 1997), la cocaína (Grimm y See, 1997; Mantsch, Ho, Schlussman y Kreek, 2001; Marinelli y White, 2000), la nicotina (Suto, Austin y Vezina, 2001) o el etanol (Nadal, Armario y Janak, 2002). Es interesante destacar que el estrés (derrota social) tiene efectos diferenciales en la adquisición de la conducta de autoadministración de cocaína en ambos tipos de poblaciones, de forma que la disminuye en los HR y la aumenta en los LR (Kabbaj, Norton, Kollack-Walker, Watson, Robinson y Akil, 2001). Sin embargo, en algunos estudios la reactividad motora se ha correlacionado con la velocidad en la adquisición de la conducta instrumental de apretar una palanca en situación operante y no propiamente con la autoadministración de la droga (Mitchell, Cunningham y Mark, 2005). Como comenta Kabbaj (2006) en su revisión, cabe mencionar también que en ciertos casos las diferencias entre las dos subpoblaciones de ratas se observan únicamente a dosis bajas, mientras que si se utilizan dosis más altas (que son normalmente más reforzantes) se diluyen las diferencias entre grupos. Además, en muchas ocasiones las subpoblaciones difieren en la adquisición inicial de la conducta de autoadministración, mientras que con el suficiente tiempo de contacto con la droga los animales LR también adquieren la conducta. Si los animales HR son en realidad más sensibles al refuerzo es una cuestión aún muy debatida. De hecho, los umbrales basales de autoestimulación eléctrica intracraneal en el haz prosencefálico medial son los mismos en los animales LR y en los HR, así como la disminución en estos umbrales que produce la anfetamina, efecto característico de las drogas de abuso (Antoniou, Papathanasiou, Panagis, Nomikos, Hyphantis y Papadopoulou-Daifoti, 2004).

¿Son en realidad las ratas HR más adictas? Un estudio más reciente del equipo de Piazza de gran impacto en el campo (Deroche-Gamonet, Belin y Piazza, 2004), ha demostrado que la reactividad motora en ambientes nuevos no está relacionada con otros índices de conducta adictiva distintos a la tasa de respuesta en los estadios iniciales de adquisición de la conducta de autoadministración, como son la autoadministración de la droga a pesar de sus consecuencias negativas (resistencia al castigo), la persistencia de la conducta en situaciones de extinción o el esfuerzo máximo que el animal está dispuesto a hacer con el objetivo de conseguir una pequeña dosis de la droga (en pruebas de razón progresiva). Por lo tanto, probablemente el rasgo de búsqueda de sensaciones aumentaría la probabilidad de que el sujeto tenga un contacto inicial con la droga, aumentaría la sensibilidad a sus efectos, afectaría la adquisición inicial de la conducta de autoadministración, pero la persistencia en el consumo estaría influenciada por otros factores. Sin embargo, desde un punto de vista preventivo pro- 
bablemente el evitar que el sujeto tenga sus primeros contactos con la droga, incide sobre la probabilidad que éste tiene de convertirse en adicto.

Respecto a la sensibilidad a los efectos reforzantes agudos de las drogas, medidas por el condicionamiento de la preferencia por un lugar, estudios iniciales indicaron que no existía relación entre el grado de actividad motora en ambientes nuevos y el desarrollo de la preferencia por cocaína (Gong, Neill y Justice, 1996; Kosten y Miserendino, 1998), anfetamina (Erb y Parker, 1994; Robinet, Rowlett y Bardo, 1998), alcohol (Cunningham, 1995) o morfina (Zheng, Ke, Tan, Luo, Xu, Yang et al., 2003). Sin embargo, datos más recientes de nuestro laboratorio indican que cuando se mide la actividad motora en ambientes nuevos durante períodos de tiempo más prolongados (valorando la perseverancia a la exploración), existe una asociación entre búsqueda de sensaciones y desarrollo de condicionamiento de la preferencia por un lugar asociado a la morfina, relación independiente del rasgo de ansiedad (Nadal, Rotllant, Márquez y Armario, 2005). Sin embargo, otros datos también recientes con cepas de ratones HEB y LEB (high-y low-exploratory behaviour) seleccionadas genéticamente por su grado de exploración en una plancha de agujeros, indican que no existe una relación fiable entre el desarrollo de condicionamiento de preferencia por un lugar asociado a la anfetamina o al etanol y la exploración en ambientes nuevos (Kliethermes, Kamens y Crabbe, 2007). Es evidente que el tema es complejo y la cuestión aún no está cerrada.

Por otra parte, los animales HR muestran una mayor reactividad a los efectos estimulantes motores de la anfetamina (Piazza et al., 1989) y de la morfina (Deroche, Piazza, Le Moal y Simon, 1993). Los resultados de otros autores apoyan los datos iniciales de este equipo, mostrando en otros trabajos los animales HR mayor sensibilidad a los efectos estimulantes motores de la anfetamina (Hooks, Jones, Smith, Neill y Justice, 1991, Hooks, Jones, Liem y Justice, 1992b; Hooks, Jones, Neill y Justice, 1992c), la cocaína (Chefer, Zakharova y Shippenberg, 2003; Gong et al., 1996; Hooks et al., 1991, 1992b; Kosten y Miserendino, 1998; Sell, Dillon, Cunningham y Thomas, 2005), la cafeína (Hooks et al., 1992b), la metamfetamina (Bevins y Peterson, 2004) y de la morfina (Kalinichev, White y Holtzman, 2004). Efectos similares se han encontrado tras la inyección tópica de dopamina y del AMPA, agonista de los aminoácidos excitadores, en el pálido ventral y el accumbens (Hooks y Kalivas, 1994; Hooks, Juncos, Jstice, Meiergerd, Povlock, Schenk et al., 1994a). Sin embargo, la mayor sensibilidad a los efectos motores de la anfetamina de las ratas HR, no parece darse a lo largo de todas las dosis, sino a dosis bajas (ver revisión en Kabbaj, 2006). Incluso en algunos estudios se ha encontrado una correlación negativa entre reactividad motora en ambientes nue- vos y efectos psicoestimulantes de la cocaína (Carey, DePalma y Damianopoulos, 2003).

Por otra parte, los animales $H R$ respecto a los LR parecen mostrar mayor sensibilización a los efectos motores de los psicoestimulantes (Hooks et al., 1991a, 1992c; Jodogne, Marinelli, Le Moal y Piazza, 1994) y del etanol (Hoshaw y Lewis, 2001), si bien los resultados varían en función de la dosis, de la cepa de ratas utilizada (ver revisión en Kabbaj, 2006), del sexo y de la edad del sujeto (Wooters, Dwoskin y Bardo, 2006).

Las diferencias individuales en reactividad motora también parecen asociadas con otros efectos del perfil farmacológico de las drogas. Por ejemplo, los animales LR presentan una mayor sensibilidad a los efectos aversivos de la anfetamina, medidas por la aversión condicionada al gusto (Kunin, Gaskin, Borjas, Smith y Amit, 2001). Este hecho es interesante puesto que anteriormente hemos comentado que los sujetos HR parecen ser más sensibles a los efectos estimulantes motores de las drogas, efectos que suelen estar relacionados con dosis que producen efectos reforzantes positivos. Por otra parte, los animales HR respecto a los LR muestran también mayores reacciones fisiológicas de abstinencia tras la retirada de un tratamiento crónico con morfina (Cecchi, Capriles, Watson y Akil, 2007). Este podría ser otro aspecto relacionado con la mayor probabilidad de desarrollar conducta adictiva mostrada por los sujetos con un alto nivel de búsqueda de sensaciones, puesto que el estado aversivo asociado a la abstinencia puede inducir recaída en el consumo.

Algunas de las diferencias que muestran las ratas HR y LR en sensibilidad a los efectos de las drogas o en sus capacidades reforzantes positivas podrían estar relacionadas con un diferente funcionamiento basal o en respuesta a las drogas de su sistema dopaminérgico. Cabe mencionar, dentro de los estudios que comentaremos posteriormente, el trabajo de Chefer et al., (2003) quien obtuvo que en respuesta a la cocaína en los HR se produce una menor recaptación de dopamina en el núcleo accumbens. Puesto que la principal acción farmacológica de la cocaína es inhibir la recaptación de dopamina, es como si la cocaína ejerciera más efecto neuroquímico en las HR que en las LR, de acuerdo también con estudios más clásicos (Hooks, Colvin, Juncos y Justice, 1992a).

\section{Reactividad al estrés y búsqueda de sensaciones}

En nuestro laboratorio hemos estudiado las diferencias individuales que existen en la respuesta al estrés, tanto agudo (García y Armario, 2001), como crónico (Márquez, Nadal y Armario, 2004). Algunos 
de los factores que modulan estas diferencias podrían ser variables de personalidad.

Un marcador prototípico de la respuesta al estrés es el eje hipotalámico-pituitario-adrenal (ver revisión en Armario, 2006). Dicho eje se inicia en la zona parvocelular del núcleo paraventricular del hipotálamo donde se encuentran los somas de las neuronas productoras de CRF o $\mathrm{CRH}$, factor liberador de corticotropina, el cual se libera en la eminencia media y viajando por el sistema portal hipofisario llega hasta la adenohipófisis o pituitaria anterior donde estimula la síntesis y liberación de ACTH u hormona adrenocorticotrópica. El ACTH viajando por el torrente circulatorio llega hasta la corteza de la glándula suprarrenal, donde estimula la síntesis y liberación de glucocorticoides como el cortisol en humanos y en otros animales como la rata, corticosterona. Los glucocorticoides ejercen un efecto de retroalimentación negativa para regular la actividad del propio eje, a nivel hipofisario, hipotalámico e incluso extrahipotalámico, puesto que existen receptores de glucocorticoides tipo GR en estructuras como el área tegmental ventral y la formación hipocámpica.

Los animales HR y LR difieren en la actividad de su eje hipotalámico-pituitario-adrenal. Por ejemplo, la inyección intravenosa de corticosterona aumenta la adquisición inicial de la autoadministración de anfetamina en los animales LR y la disminuye en los HR (Piazza et al., 1991). Los sujetos LR y HR también difieren en la autoadministración de corticosterona, los primeros muestran un desplazamiento hacia la derecha de la curva dosis-respuesta de autoadministración, indicando que el poder reforzante de ésta es menor en los animales LR (Piazza, Deroche, Deminière, Maccari, LeMoal y Simon, 1993). Es decir, es como si la corticosterona que se libera por ejemplo en situaciones de estrés fuera reforzante para los animales HR. Además, la reactividad motora a la morfina potenciada en los sujetos HR desaparece cuando éstos son sometidos a una adrenalectomía (y suplementados a nivel basal con corticosterona), con el objetivo de suprimir el incremento en los niveles de corticosterona producido en situaciones de estrés (Deroche et al., 1993).

La reactividad al estrés también parece ser distinta en los animales HR respecto a los LR. En el estudio de Piazza et al (1991), las ratas HR presentaron unos mayores niveles de corticosterona tras $2 \mathrm{~h}$ de exposición en el corredor circular respecto a las ratas $L R$. En estudios posteriores (Bouyer, Vallee, Deminière, Le Moal y Mayo, 1998; Dellu, Mayo, Vallee, Maccari, Piazza, Le Moal et al, 1996a) se encontró también que la recuperación de los niveles de corticosterona tras la finalización de un estímulo estresante de mayor intensidad (inmovilización en tubo) fue más lenta en los animales HR que en los LR. La respuesta al estrés en los animales HR es también mayor que la de los LR a nivel de la liberación de dopamina en el accumbens (Rougé-Pont, Piazza, Kharouby, Le Moal y Simon,
1993), diferencia que es anulada por la adrenalectomía (Rougé-Pont, Deroche, Le Moal y Piazza, 1998).

Sin embargo, en otros trabajos (Kabbaj et al., 2000), la recuperación de los niveles plasmáticos de corticosterona tras la finalización de un ambiente nuevo (prueba de luz-oscuridad) era más lenta en los animales HR que en los LR, mientras que ambas subpoblaciones no diferían cuando el estímulo estresante era de mayor intensidad (inmovilización en tubo). Estos autores discuten que el grado de control que el animal ejerce sobre la situación puede ser un factor crítico en estas diferencias, de manera que no se manifestarían cuando el estímulo estresante está "impuesto" por el investigador. Sin embargo, ambos estímulos (inmovilización en tubo y prueba de luz-oscuridad) no únicamente difieren en el grado de control que pueda ejercer el animal, sino también en la intensidad del estímulo estresante. En nuestro laboratorio (Márquez, Nadal y Armario, 2006), hemos comparado la respuesta plasmática de ACTH (hormona que no se ha estudiado en otros trabajos) y de corticosterona ante dos tipos de estímulos estresantes de intensidad leve, dos ambientes nuevos que difieren en el grado de controlabilidad que el animal ejerce sobre la situación, la prueba de luz-oscuridad (el sujeto puede permanecer en el compartimiento oscuro poco estresante) y la plancha de agujeros (es un ambiente más inescapable). En ambos casos hemos encontrado que los animales HR presentan una mayor respuesta hormonal y una recuperación más lenta de los niveles basales tras la finalización del estímulo estresante. Por tanto, al menos en este caso no sería el factor crítico el grado de control, sino la intensidad del estrés.

Sin embargo en otros estudios, la relación entre reactividad motora en ambientes nuevos y niveles plasmáticos de corticosterona en respuesta a ambientes nuevos ha sido negativa (Cavigelli y McClintock, 2003; Shimosato y Watanabe, 2003) o bien no se ha hallado diferencia alguna (Wrona, Jurkowski, Luszawska, Tokarski y Trojniar, 2003; Wrona, Jurkowski y Tokarski, 2004)

Muchas drogas de abuso producen también una activación del eje hipotalámico-pituitario-adrenal, de forma que se podrían considerar éstas como estímulos estresantes. Apenas existen estudios sobre la relación entre actividad motora en ambientes nuevos y respuesta plasmática hormonal ante las drogas. En el único estudio que conocemos, Wrona, Sukiennik, Jurkowski, Jurkowlaniec, Glac y Tokarski (2005) obtuvieron que la respuesta plasmática a nivel de corticosterona ante la anfetamina era mayor en las ratas HR que en las LR. En nuestro laboratorio, estudios recientes (Gagliano et al., en preparación) apoyan el hecho de que los animales HR muestran una mayor respuesta a la anfetamina a nivel de los niveles plasmáticos de corticosterona y además estos niveles plasmáticos tardan más en recuperarse. Esta diferencia entre los 
sujetos HR y LR mostrada a nivel agudo se mantiene tras administraciones repetidas de la droga; es decir, parece ser un rasgo individual consistente.

La diferente reactividad del eje hipotalámico-pituitario-adrenal a ciertos estímulos estresantes que presentan los animales HR y LR se ve apoyada como comentaremos posteriormente por la existencia de mayores niveles de CRF en el núcleo paraventricular del hipotálamo en los sujetos HR junto con una menor expresión de receptores de glucocorticoides tipo GR en la formación hipocampal, como muestra de un supuesto menor funcionamiento de los mecanismos de retroinhibición del eje hipotalámico-pituitario-adrenal (Kabbaj, Devine, Savage y Akil, 2000). Además, los animales HR muestran una mayor activación neural (medida por la expresión del gen c-fos) en el núcleo paraventricular en respuesta a un estrés de intensidad leve, como es la exposición a un ambiente nuevo (Kabbaj y Akil, 2001).

\section{Substrato neurobiológico de las diferencias individua-} les en la búsqueda de sensaciones

El substrato neurobiológico de las diferencias individuales parece residir en el sistema dopaminérgico, que ha sido uno de los más estudiados en su relación con la adicción (ver Corominas-Rosó, Roncero, Bruguera y Casas, 2007 para una revisión) y en el eje hipotalámico-pituitario-adrenal, que es uno de los sistemas implicados en la respuesta al estrés (ver Armario, 2006 para una revisión). Los estudios sobre el tema se han realizado bien en condiciones basales, o bien tras la administración de un psicoestimulante, que es el tipo de droga que más se ha estudiado en relación a la búsqueda de sensaciones. Respecto al sistema dopaminérgico, la mayoría de los estudios se han centrado en la vía mesocorticolímbica que se origina en el área tegmental ventral y finaliza en zonas de la corteza (básicamente prefrontal) y del sistema límbico (como el accumbens o la amígdala), y en la vía negroestriada que se origina en la substancia negra y finaliza en el estriado dorsal. Puesto que psicoestimulantes como la anfetamina y la cocaína ejercen sus efectos neuroquímicos principalmente sobre el transportador de dopamina, inhibiendo la recaptación de dicho neurotransmisor, se ha investigado el papel de dicho transportador, así como de los receptores dopaminérgicos en el rasgo de búsqueda de sensaciones.

En el sistema dopaminérgico, los animales HR respecto a los LR presentan mayor:

- Nivel basal de receptores de dopamina tipo D1 en el accumbens, medidos por técnicas de radioligando (Hooks, Sorg y Kalivas, 1994b).
- Tasa basal de descarga de neuronas dopaminérgicas del área tegmental ventral y de la substancia negra (Marinelli y White, 2000).

- Incremento en la concentración extracelular de dopamina en el accumbens en respuesta a la cocaína, estudiado mediante microdiálisis (Chefer et al., 2003).

- Nivel del transportador de dopamina en el área tegmental ventral y en la substancia negra, tras la inyección repetida de anfetamina, medidos mediante hibridación in situ (Dietz, Tapocik, GavalCruz y Kabbaj, 2005).

También los animales HR presentan mayor:

- Nivel basal de CRF en el núcleo paraventricular del hipotálamo, medidos por hibridación in situ (Kabbaj et al., 2000)

- Expresión de c-fos tras la exposición a un ambiente nuevo en el núcleo paraventricular del hipotálamo, medidos por hibridación in situ (Kabbaj y Akil, 2001).

- Nivel basal de pre-pro-encefalina y de dinorfina en el accumbens shell, medidos por hibridación in situ (Lucas, Angulo, Le Moal, McEwen y Piazza, 1998).

- Nivel basal de noradrenalina en el tálamo y la amígdala, y de serotonina en la amígdala (Ray, Hansen y Waters, 2006).

En cambio, en referencia también al sistema dopaminérgico, los sujetos HR respecto a los LR presentan menor:

- Nivel basal de receptores de dopamina tipo D2 en el accumbens y el estriado dorsal, medidos por técnicas de radioligando (Hooks et al., 1994b).

- Nivel basal del principal enzima regulador de la síntesis de las catecolaminas, la tirosina hidroxilasa en el área tegmental ventral, medidos por radio-inmuno-citoquímica (Lucas et al., 1998).

- Sensibilidad de las neuronas dopaminérgicas de el área tegmental ventral y de la substancia negra al efecto inhibitorio en la tasa de descarga producido por los agonistas de dopamina tipo D2 (Marinelli y White, 2000).

- Recaptación de dopamina, tanto a nivel basal como tras la administración de cocaína, medida por técnicas de microdiálisis (Chefer et al., 2003).

- Proporción basal del receptor de dopamina tipo D3 respecto a una variante de la proteína que no se une a la dopamina (D3nf) en la corteza prefrontal y en la substancia negra/área tegmental ventral, 
medidas por técnicas de RT-PCR (Pritchard, Logue, Taylor, Ahlbrand, Welge, Tang et al., 2006).

- Nivel basal de dopamina en el tronco del encéfalo (Ray et al., 2006).

También los animales HR presentan menor:

- Nivel basal de neurotensina en el accumbens shell, medidos por Northern-blot (Hooks et al., 1994a).

- Nivel basal de colecistoquinina en el área tegmental ventral y en la substancia negra, medidos por hibridación in situ (Lucas et al., 1998).

- Nivel basal de CRF en el núcleo central de la amígdala y de receptores de glucocorticoides tipo GR en la formación hipocámpica, medidos por hibridación in situ (Kabbaj et al., 2000).

- Nivel basal de serotonina en el tronco del encéfalo y del metabolito de la serotonina (ácido hidroxiindol-acético) en la amígdala (Ray et al., 2006).

En cambio, a nivel del sistema dopaminérgico, no presentan diferencias los animales HR versus los LR en:

- Nivel basal del transportador de dopamina en la substancia negra, medidos por Northern-blot (Hooks et al., 1994b).

- Nivel basal de la tirosina hidroxilasa en la substancia negra, en el caudado-putamen y en el accumbens, medidos por radio-inmuno-citoquímica (Lucas et al., 1998).

- Nivel basal de la tirosina hidroxilasa en el área tegmental ventral, medidos por hibridación in situ (Lucas et al., 1998).

- Concentración extracelular a nivel basal de dopamina en el accumbens, medida mediante técnicas de microdiálisis (Chefer et al., 2003).

- Nivel basal del transportador de dopamina en sinaptosomas del accumbens, medidos por inmunoblots (Chefer et al., 2003).

- Nivel basal del transportador de dopamina en la substancia negra y el área tegmental ventral, medidos por hibridación in situ (Dietz et al., 2005).

El rasgo de búsqueda de sensaciones y su independencia con la ansiedad

Tanto en los estudios de los equipos de Le Moal y Piazza (Dellu et al., 1993), como en los de Akil (Kabbaj et al., 2000), los animales outbred HR y LR no únicamente difieren en la reactividad motora en ambientes nuevos, sino también en medidas de ansiedad.
Lo mismo sucede en las ratas RHA y RLA (Steimer y Driscoll, 2003) y en las cepas de ratas HR-bred y LRbred seleccionadas genéticamente por el laboratorio de Huda Akil por su nivel de actividad en ambientes nuevos (Stead, Clinton, Neal, Schneider, Jama, Miller et al., 2006). De esta manera resulta difícil diferenciar el papel aislado de ambos rasgos (ansiedad y búsqueda de sensaciones) en la reactividad al estrés y la vulnerabilidad a la adicción.

En las condiciones experimentales de nuestro laboratorio con ratas outbred no seleccionadas (Márquez et al., 2006; Nadal et al., 2005) la reactividad motora en ambientes nuevos (valorada en un corredor circular) es independiente de la ansiedad (valorada en un laberinto elevado en forma de cruz). Tanto la respuesta hormonal del eje hipotalámico-pituitarioadrenal al estrés que representa un ambiente nuevo (Márquez et al., 2006) como el desarrollo del condicionamiento de la preferencia por un lugar (Nadal et al., 2005) están relacionadas con la reactividad motora en una situación inescapable, mientras que las medidas de ansiedad son independientes. En otros laboratorios (Abreu-Villaça, Queiroz-Gomes, Dal Monte, Filgueiras y Manhaes, 2006) también se ha podido establecer en ratones que la exploración en una plancha de agujeros está relacionada con la preferencia oral por una solución de nicotina, mientras que las medidas de ansiedad son independientes de dicha preferencia.

\section{Modelos de búsqueda de sensaciones en situación de libre elección}

En el laboratorio de Michael Bardo se describió que la preferencia por ambientes nuevos no predice la respuesta motora a la anfetamina, mientras que en el mismo trabajo se encontró que la actividad en ambientes nuevos inescapables si que está relacionada con la sensibilidad a los efectos estimulantes de la droga (Robinet et al., 1998), de acuerdo con los trabajos comentados anteriormente. La exploración de objetos nuevos predice el desarrollo de condicionamiento de la preferencia por un lugar asociado a la anfetamina (Klebaur y Bardo, 1999) pero no la autoadministración operante de la droga (Klebaur, Bevins, Segar y Bardo, 2001). Sin embargo, en un modelo de regresión múltiple si se incluyen en el análisis tanto medidas de reactividad motora en ambientes inescapables como medidas de preferencia por la novedad, se obtiene una mejor predicción del grado de autoadministración operante de anfetamina que si se incluye en el modelo únicamente la reactividad en ambientes inescapables (Cain, Saucier y Bardo, 2005). En otro estudio reciente, la preferencia por la novedad también se ha relacionado con el desarrollo de condicionamiento de la 
preferencia por un lugar a la morfina en ratas outbred (Pelloux, Costentin y Duterte-Boucher, 2006).

En un modelo animal con "mini-cerdos", utilizando técnicas de PET, Lind, Gjedde, Moustgaard, Olsen, Jensen y Jakobsen (2005) señalan que la exploración de objetos nuevos correlaciona positivamente con la reducción que ejerce la anfetamina en la unión del raclopride, antagonista de los receptores de la dopamina tipo D2/D3, en el estriado ventral izquierdo, indicando que el rasgo de búsqueda de sensaciones está relacionado con un mayor aumento de los niveles de dopamina producido por la anfetamina. Es interesante destacar que dicha relación se obtenía únicamente en animales macho y no en hembras. Cabe recordar también que estos resultados concuerdan con los datos de Chefer et al., (2003) que señalan que en ratas la actividad motora en un ambiente inescapable está relacionada con un mayor aumento en los niveles extracelulares de dopamina en el accumbens, medidos mediante técnicas de microdiálisis, en respuesta a la cocaína. Asimismo estos resultados concuerdan también con los comentados posteriormente en humanos (Leyton, Boileau, Benkelfat, Diksic, Baker y Dagher, 2002).

La relación entre preferencia por la novedad y niveles de dopamina en el accumbens podría estar afectada por la edad del sujeto. Stansfield y Kirstein (2005) mostraron en ratas outbred mediante técnicas de microdiálisis que la proporción entre los niveles extracelulares de dopamina en el accumbens tras la inyección de cocaína respecto a los niveles tras la inyección de salino era más elevada en los animales con mayor grado de exploración de objetos nuevos, solo en el caso de animales adolescentes, dándose la relación contraria en animales adultos.

La exploración de objetos nuevos también parece estar relacionada con el receptor de dopamina tipo D4, puesto que los ratones $\mathrm{KO}$ del receptor muestran una disminución en la exploración de estímulos nuevos (Dulawa, Grandy, Low, Paulus y Geyer, 1999) y se ha descrito también un polimorfismo en el receptor D4 similar al descrito en humanos (ver apartado siguiente) asociado con la exploración de objetos nuevos en monos (Bailey, Breidenthal, Jorgensen, McCracken y Fairbanks, 2007).

\section{ESTUDIOS EN HUMANOS}

Diversos estudios retrospectivos han asociado búsqueda de sensaciones e impulsividad con adicción (Acton, 2003; Barrón Pardo, Mateos Agut y Martínez Villate, 2004; Billieux, Van der Linden y Ceschi, 2007; Conway, Kane, Ball, Poling y Rounsaville 2003; Franques, Auriacombe, Piquemal, Verger, Brisseau-
Gimenez, Grabot et al., 2003; Le Bon, Basiaux, Streel, Tecco, Hanak, Hansenne et al., 2004; Sher, Bartholow y Wood, 2000; Wills, Vaccaro y McNamara, 1994). Además, en los últimos años, han aparecido estudios experimentales en situación de laboratorio en humanos que a grandes rasgos apoyan los datos en animales, a pesar de que el tema es complejo, no está resuelto y las relaciones son sutiles.

Los estudios de laboratorio realizados hasta el momento han utilizado la anfetamina, demostrando que esta droga produce más efecto conductual y subjetivo en los individuos con alto nivel de búsqueda de sensaciones (Fleming, Bigelow, Weinberger y Goldberg, 1995; Hutchison, Wood y Swift, 1999; Kelly, Robbins, Martin, Fillmore, Lane, Harrington et al., 2006; Sax y Strakowski, 1998), si bien ciertos datos no apoyan esta relación (Corr y Kumasi, 2000; White, Lott y de Wit, 2006b). La capacidad reforzante de la droga ha sido evaluada mediante una tarea de razón progresiva, encontrándose que los sujetos con elevado nivel de búsqueda de sensaciones son capaces de realizar un mayor número de respuestas operantes seguidas para conseguir como refuerzo una única dosis de la droga que los sujetos de niveles bajos (Stoops, Lile, Robbins, Martin, Rush y Kelly, 2007).

Existe poca información sobre la relación entre reactividad al estrés y búsqueda de sensaciones en humanos. Oswald, Mathena y Wand (2004) obtuvieron que en una tarea de estrés social y aritmético existía una correlación positiva entre el grado de extraversión (incluyendo componentes de búsqueda de sensaciones) y los niveles plasmáticos de ACTH, si bien esta relación puede ser exclusiva del género masculino (Oswald, Zandi, Nestadt, Potash, Kalaydjian y Wand, 2006). Otros estudios apoyan esta relación positiva entre eje hipotalámico-pituitario-adrenal y búsqueda de sensaciones (Harl, Weisshuhn y Kerschbaum, 2006), si bien otros datos sugieren una relación negativa entre ambos (Tyrka, Wier, Anderson, Wilkinson, Price y Carpenter, 2007). No conocemos tampoco si en humanos esta dimensión de personalidad se asocia con una mayor respuesta del eje hipotalámico-pituitario-adrenal ante la administración de drogas de abuso, pero en el estudio de White, Grover y de Wit (2006a) se obtuvo que el pico en los niveles de cortisol que se obtienen tras la inyección de anfetamina estaba avanzado en los sujetos con un elevado rasgo de búsqueda de sensaciones.

Otra cuestión muy interesante a nivel teórico es si la búsqueda de sensaciones está asociada con un mayor efecto neuroquímico de las drogas de abuso. Así parece ser puesto que en el estudio de Leyton et al., (2002) se obtuvo que tras la administración de anfetamina, se obtiene una correlación positiva entre la búsqueda de sensaciones y los niveles extracelu- 
lares de dopamina en el estriado ventral (medidos en estudios de tomografía de emisión de positrones por el desplazamiento que se produce en la unión del radioligando raclopride, antagonista de los receptores de dopamina tipo D2/D3). En este mismo estudio se encontró que los niveles extracelulares de dopamina también correlacionaban positivamente con el deseo por la droga manifestado por los sujetos en una escala análoga visual, y este deseo estaba relacionado a su vez con ciertos componentes del rasgo de búsqueda de novedad. Además, el incremento en los niveles extracelulares de dopamina en el estriado ventral tras la inyección repetida de anfetamina se sensibiliza, y esta sensibilización correlaciona positivamente con la búsqueda de sensaciones (Boileau, Dagher, Leyton, Gunn, Baker, Diksic et al., 2006). Por otra parte, los niveles de cortisol tras la inyección aguda de anfetamina correlacionan positivamente con el incremento en los niveles de dopamina extracelular que produce la droga en el estriado ventral y con la sensación de euforia y bienestar desencadenada por la administración de la droga (Oswald, Wong, McCaul, Zhou, Kuwabara, Choi et al., 2005). Los niveles de cortisol ante un estrés psicosocial también se han relacionado con la liberación de dopamina en el estriado ventral inducida por la anfetamina (Wand, Oswald, McCaul, Wong, Johnson, Zhou et al., 2007) y con la recaída en el consumo de cocaína tras una etapa de abstinencia (Sinha, Garcia, Paliwal, Kreek y Rounsaville, 2006). Estos hechos junto con los datos descritos en animales sugieren que la respuesta del eje hipotalámico-pituitario-adrenal a ciertos estímulos estresantes podría ser un posible marcador de vulnerabilidad a la adicción.

Otra de las pruebas que sugieren que existe una relación entre el sistema dopaminérgico, la búsqueda de sensaciones y la adicción, es la posible existencia de un polimorfismo del receptor de dopamina tipo D4, polimorfismo que se asocia con una mayor funcionalidad del receptor, en los sujetos con un elevado nivel de búsqueda de sensaciones (Ebstein, Novick, Umansky, Priel, Osher, Blaine et al., 1996). Posteriormente este dato fue replicado en algunos estudios pero no en otros, apoyando la idea de que la relación es sutil y que la genética del rasgo es compleja (Lusher, Chandler y Ball, 2001; Savitz y Ramesar, 2004; Ebstein, 2006).

\section{CONCLUSIONES}

Dentro de la multiplicidad de factores de base genética y ambiental que pueden afectar la vulnerabilidad a las drogas, los rasgos de personalidad pueden desempeñar un papel muy relevante. Una de las dimensiones de personalidad que más se ha relacionado en animales y en humanos con la susceptibilidad a la adicción ha sido el de búsqueda de sensaciones y/o novedades (novelty-seeking). En modelos animales dicho rasgo se ha evaluado principalmente mediante la medida de la actividad motora/exploración en ambientes nuevos. Parece ser que tanto en animales como en humanos dicho rasgo se asocia con una mayor sensibilidad a ciertos efectos de las drogas, con una mayor facilidad para adquirir (especialmente de forma inicial) la conducta adictiva, con un mayor efecto neuroquímico de las drogas (al menos psicoestimulantes) a nivel del sistema dopaminérgico y también con una mayor reactividad ante ciertas situaciones estresantes a nivel del eje hipotalámico-pituitario-adrenal. Puesto que el estrés es un factor que se ha relacionado con la conducta adictiva, estos hechos pueden tener interesantes repercusiones preventivas y/o terapéuticas. Si la respuesta del eje hipotalámico-pituitario-adrenal ante ciertos estímulos estresantes es un marcador de vulnerabilidad a la adicción viene avalado por ciertos datos pero tiene que ser evaluado en futuros estudios.

\section{AGRADECIMIENTOS}

Red de trastornos adictivos (Redes temáticas, Instituto de Salud Carlos III, Ministerio de Sanidad y Consumo), Plan Nacional sobre drogas (Ministerio de Sanidad y Consumo) y DGICYT SAF2005-00358 (Ministerio de Educación y Ciencia).

\section{REFERENCIAS}

Abreu-Villaca, Y., Queiroz-Gomes Fdo, E., Dal Monte, A. P., Filgueiras, C. C., y Manhaes, A. C. (2006). Individual differences in novelty-seeking behavior but not in anxiety response to a new environment can predict nicotine consumption in adolescent C57BL/6 mice. Behavioural Brain Research, 167, 175-182.

Acton, G.S (2003) Measurement of impulsivity in a hierarchical model of personality traits: implications for substance use. Substance Use and Misuse, 38, 67-83.

Antoniou, K., Papathanasiou, G., Panagis, G., Nomikos, G. G., Hyphantis, T., y Papadopoulou-Daifoti, Z. (2004). Individual responses to novelty predict qualitative differences in d-amphetamine-induced open field but not reward-related behaviors in rats. Neuroscience, 123, 613-623.

Armario, A. (2006). The hypothalamic-pituitary-adrenal axis: what can it tell us about stressors? CNS Neurological Disorders Drug Targets, 5, 485-501.

Bailey, J. N., Breidenthal, S. E., Jorgensen, M. J., McCracken, J. T., y Fairbanks, L. A. (2007). The association of DRD4 
and novelty seeking is found in a nonhuman primate model. Psychiatric Genetics, 17, 23-27.

Bardo, M. T., Donohew, R. L., y Harrington, N. G. (1996). Psychobiology of novelty seeking and drug seeking behavior. Behavioural Brain Research, 77, 23-43.

Barron Pardo, E., Mateos Agut, M. y Martínez Villate, I. (2004) Temperamento y carácter en adictos a cocaína y a opiáceos. Adicciones, 16, 287-294.

Bevins, R. A., y Peterson, J. L. (2004). Individual differences in rats' reactivity to novelty and the unconditioned and conditioned locomotor effects of methamphetamine. Pharmacology, Biochemistry and Behavior, 79, 65-74.

Billieux, J., Van der Linden, M., y Ceschi, G. (2007). Which dimensions of impulsivity are related to cigarette craving? Addictive Behavior, 32, 1189-1199.

Boileau, I., Dagher, A., Leyton, M., Gunn, R. N., Baker, G. B., Diksic, M., et al., (2006). Modeling sensitization to stimulants in humans: an [11C]raclopride/positron emission tomography study in healthy men. Archives of General Psychiatry, 63, 1386-1395.

Bouyer, J. J., Vallee, M., Deminière, J. M., Le Moal, M., y Mayo, W. (1998). Reaction of sleep-wakefulness cycle to stress is related to differences in hypothalamopituitary-adrenal axis reactivity in rat. Brain Research, 804, 114-124.

Cabib, S., Orsini, C., Le Moal, M., y Piazza, P. V. (2000). Abolition and reversal of strain differences in behavioral responses to drugs of abuse after a brief experience. Science, 289, 463-465.

Cain, M. E., Denehy, E. D., y Bardo, M. T. (2007). Individual Differences in Amphetamine Self-Administration: The Role of the Central Nucleus of the Amygdala. Neuropsychopharmacology, en prensa.

Cain, M. E., Saucier, D. A., y Bardo, M. T. (2005). Novelty seeking and drug use: contribution of an animal model. Experimental and Clinical Psychopharmacology, 13, 367-375.

Carey, R. J., DePalma, G., y Damianopoulos, E. (2003). Response to novelty as a predictor of cocaine sensitization and conditioning in rats: a correlational analysis. Psychopharmacology, 168, 245-252.

Cavigelli, S. A., y McClintock, M. K. (2003). Fear of novelty in infant rats predicts adult corticosterone dynamics and an early death. Proceedings of the National Academy of Sciences U S A, 100, 16131-16136.

Cecchi, M., Capriles, N., Watson, S. J., y Akil, H. (2007). Beta1 adrenergic receptors in the bed nucleus of stria terminalis mediate differential responses to opiate withdrawal. Neuropsychopharmacology, 32, 589-599.

Chefer, V. I., Zakharova, I., y Shippenberg, T. S. (2003). Enhanced responsiveness to novelty and cocaine is associated with decreased basal dopamine uptake and release in the nucleus accumbens: quantitative microdialysis in rats under transient conditions. Journal of Neuroscience, 23, 3076-3084.

Cloninger, C. R. (1994). Temperament and personality. Current Opinion in Neurobiology, 4, 266-273.
Cloninger, C. R., Sigvardsson, S. y Bohman, M. (1988). Childhood personality predicts alcohol abuse in young adults. Alcoholism, Clinical and Experimental Research, 12, 494-505.

Conway, K. P., Kane, R. J., Ball, S. A., Poling, J. C., y Rounsaville, B. J. (2003). Personality, substance of choice, and polysubstance involvement among substance dependent patients. Drug and Alcohol Dependence, 71, 65-75.

Corominas-Rosó, M., Roncero, C., Bruguera, E., y Casas, M. (2007) Sistema dopaminérgico y adicciones. Revista de Neurología, 44, 23-31.

Corr, P. J., y Kumari, V. (2000). Individual differences in mood reactions to d-amphetamine: a test of three personality factors. Journal of Psychopharmacology, 14, 371-377.

Cunningham, C. L. (1995). Localization of genes influencing ethanol-induced conditioned place preference and locomotor activity in BXD recombinant inbred mice. Psychopharmacology, 120, 28-41.

Dellu, F., Mayo, W., Piazza, P. V., Le Moal, M., y Simon, H. (1993). Individual differences in behavioural response to novelty in rats. Possible relationship with the sensationseeking trait in man. Personality and Individual Differences, 15, 411-418.

Dellu, F., Mayo, W., Vallee, M., Maccari, S., Piazza, P. V., Le Moal, M., et al., (1996a). Behavioral reactivity to novelty during youth as a predictive factor of stress-induced corticosterone secretion in the elderly-a life-span study in rats. Psychoneuroendocrinology, 21, 441-453.

Dellu, F., Piazza, P. V., Mayo, W., Le Moal, M., y Simon, H. (1996b). Novelty-seeking in rats- biobehavioral characteristics and possible relationship with the sensation-seeking trait in man. Neuropsychobiology, 34, 136-145.

Deroche, V., Piazza, P. V., Le Moal, M., y Simon, H. (1993). Individual differences in the psychomotor effects of morphine are predicted by reactivity to novelty and influenced by corticosterone secretion. Brain Research, $623,341-344$.

Deroche-Gamonet, V., Belin, D., y Piazza, P. V. (2004). Evidence for addiction-like behavior in the rat. Science, 305, 1014-1017.

Dietz, D. M., Tapocik, J., Gaval-Cruz, M., y Kabbaj, M. (2005). Dopamine transporter, but not tyrosine hydroxylase, may be implicated in determining individual differences in behavioral sensitization to amphetamine. Physiology and Behavior, 86, 347-355.

Dulawa, S. C., Grandy, D. K., Low, M. J., Paulus, M. P., y Geyer, M. A. (1999). Dopamine D4 receptor-knockout mice exhibit reduced exploration of novel stimuli. Journal of Neuroscience, 19, 9550-9556.

Ebstein, R. P. (2006). The molecular genetic architecture of human personality: beyond self-report questionnaires. Molecular Psychiatry, 11, 427-445.

Ebstein, R. P., Novick, O., Umansky, R., Priel, B., Osher, Y., Blaine, D., et al., (1996). Dopamine D4 receptor (D4DR) exon III polymorphism associated with the human 
personality trait of Novelty Seeking. Nature Genetics, 12, 78-80.

Ellenbroek, B. A., van der Kam, E. L., van der Elst, M. C., y Cools, A. R. (2005). Individual differences in drug dependence in rats: the role of genetic factors and life events. European Journal of Pharmacology, 526, 251-258.

Erb, S. M., y Parker, L. A. (1994). Individual differences in novelty-induced activity do not predict strength of amphetamine-induced place conditioning. Pharmacology, Biochemistry and Behavior, 48, 581-586.

Fleming, K., Bigelow, L. B., Weinberger, D. R., y Goldberg, T. E. (1995). Neuropsychological effects of amphetamine may correlate with personality characteristics. Psychopharmacoly Bulletin, 31, 357-362.

Franques, P., Auriacombe, M., Piquemal, E., Verger, M., Brisseau-Gimenez, S., Grabot, D., et al., (2003). Sensation seeking as a common factor in opioid dependent subjects and high risk sport practicing subjects. A cross sectional study. Drug and Alcohol Dependence, 69, 121-126.

Garcia, A., y Armario, A. (2001). Individual differences in the recovery of the hypothalamic-pituitary-adrenal axis after termination of exposure to a severe stressor in outbred male Sprague-Dawley rats. Psychoneuroendocrinology, 26, 363-374.

Gong, W., Neill, D. B., y Justice, J. B., Jr. (1996). Locomotor response to novelty does not predict cocaine place preference conditioning in rats. Pharmacology, Biochemistry and Behavior, 53, 191-196.

Grimm, J. W., y See, R. E. (1997). Cocaine self-administration in ovariectomized rats is predicted by response to novelty, attenuated by 17-beta estradiol, and associated with abnormal vaginal cytology. Physiology and Behavior, 61, 755-761.

Harl, B., Weisshuhn, S., y Kerschbaum, H. H. (2006). Cortisol titre increases with novelty of academic oral examinations. Neuro Endocrinology Letters, 27, 669-674.

Hooks, M. S., Colvin, A. C., Juncos, J. L., y Justice, J. B., Jr. (1992a). Individual differences in basal and cocainestimulated extracellular dopamine in the nucleus accumbens using quantitative microdialysis. Brain Research, 587, 306-312.

Hooks, M. S., Jones, G. H., Liem, B. J., y Justice, J. B., Jr. (1992b). Sensitization and individual differences to IP amphetamine, cocaine, or caffeine following repeated intracranial amphetamine infusions. Pharmacology, Biochemistry and Behavior, 43, 815-823.

Hooks, M. S., Jones, G. H., Neill, D. B., y Justice, J. B., Jr. (199c2). Individual differences in amphetamine sensitization: dose-dependent effects. Pharmacology, Biochemistry and Behavior, 41, 203-210.

Hooks, M. S., Jones, G. H., Smith, A. D., Neill, D. B., y Justice, J. B., Jr. (1991). Individual differences in locomotor activity and sensitization. Pharmacology, Biochemistry and Behavior, 38, 467-470.
Hooks, M. S., Juncos, J. L., Justice, J. B., Jr., Meiergerd, S. M., Povlock, S. L., Schenk, J. O., et al., (1994a). Individual locomotor response to novelty predicts selective alterations in D1 and D2 receptors and mRNAs. Journal of Neuroscience, 14, 6144-6152.

Hooks, M. S., y Kalivas, P. W. (1994). Involvement of dopamine and excitatory amino acid transmission in novelty-induced motor activity. Journal of Pharmacology and Experimental Therapeutics, 269, 976-988.

Hooks, M. S., Sorg, B. A., y Kalivas, P. W. (1994b). The relationship between MRNA levels and the locomotor response to novelty. Brain Research, 663, 312-316.

Hoshaw, B. A., y Lewis, M. J. (2001). Behavioral sensitization to ethanol in rats: evidence from the Sprague-Dawley strain. Pharmacology, Biochemistry and Behavior, 68, 685-690.

Hutchison, K. E., Wood, M. D., y Swift, R. (1999). Personality factors moderate subjective and psychophysiological responses to d-amphetamine in humans. Experimental and Clinical Psychopharmacology, 7, 493-501.

Jodogne, C., Marinelli, M., Le Moal, M., y Piazza, P. V. (1994). Animals predisposed to develop amphetamine selfadministration show higher susceptibility to develop contextual conditioning of both amphetamine-induced hyperlocomotion and sensitization. Brain Research, 657, 236-244.

Kabbaj, M. (2004). Neurobiological bases of individual differences in emotional and stress responsiveness: high responders-low responders model. Archives of Neurology, 61, 1009-1012.

Kabbaj, M. (2006). Individual differences in vulnerability to drug abuse: the high responders/low responders model. CNS Neurological Disorders Drug Targets, 5, 513-520.

Kabbaj, M., y Akil, H. (2001). Individual differences in noveltyseeking behavior in rats: a c-fos study. Neuroscience, 106, 535-545.

Kabbaj, M., Devine, D. P., Savage, V. R., y Akil, H. (2000). Neurobiological correlates of individual differences in novelty-seeking behavior in the rat: differential expression of stress-related molecules. Journal of Neuroscience, 20, 6983-6988.

Kabbaj, M., Norton, C. S., Kollack-Walker, S., Watson, S. J., Robinson, T. E., y Akil, H. (2001). Social defeat alters the acquisition of cocaine self-administration in rats: role of individual differences in cocaine-taking behavior. Psychopharmacology, 158, 382-387.

Kalinichev, M., White, D. A., y Holtzman, S. G. (2004). Individual differences in locomotor reactivity to a novel environment and sensitivity to opioid drugs in the rat. I. Expression of morphine-induced locomotor sensitization. Psychopharmacology, 177, 61-67.

Kelly, T. H., Robbins, G., Martin, C. A., Fillmore, M. T., Lane, S. D., Harrington, N. G., et al., (2006). Individual differences in drug abuse vulnerability: d-amphetamine and sensation-seeking status. Psychopharmacology, 189, 17-25. 
Klebaur, J. E., y Bardo, M. T. (1999). Individual differences in novelty seeking on the playground maze predict amphetamine conditioned place preference. Pharmacology, Biochemistry and Behavior, 63, 131-136.

Klebaur, J. E., Bevins, R. A., Segar, T. M., y Bardo, M. T. (2001). Individual differences in behavioral responses to novelty and amphetamine self-administration in male and female rats. Behavioral Pharmacology, 12, 267-275.

Kliethermes, C. L., Kamens, H. M., y Crabbe, J. C. (2007). Drug reward and intake in lines of mice selectively bred for divergent exploration of a hole board apparatus. Genes, Brain and Behavior, en prensa.

Kosten, T. A., y Miserendino, M. J. (1998). Dissociation of novelty- and cocaine-conditioned locomotor activity from cocaine place conditioning. Pharmacology, Biochemistry and Behavior, 60, 785-791.

Kunin, D., Gaskin, S., Borjas, M. B., Smith, B. R., y Amit, Z. (2001). Differences in locomotor response to an inescapable novel environment predict sensitivity to aversive effects of amphetamine. Behavioral Pharmacology, 12, 61-67.

Laviola, G., Macri, S., Morley-Fletcher, S., y Adriani, W. (2003). Risk-taking behavior in adolescent mice: psychobiological determinants and early epigenetic influence. Neuroscience and Biobehavioral Reviews, 27, 19-31.

Le Bon, O., Basiaux, P., Streel, E., Tecco, J., Hanak, C., Hansenne, M., et al., (2004). Personality profile and drug of choice; a multivariate analysis using Cloninger's TCl on heroin addicts, alcoholics, and a random population group. Drug and Alcohol Dependence, 73, 175-182.

Leyton, M., Boileau, I., Benkelfat, C., Diksic, M., Baker, G., y Dagher, A. (2002). Amphetamine-induced increases in extracellular dopamine, drug wanting, and novelty seeking: a PET/[11C]raclopride study in healthy men. Neuropsychopharmacology, 27, 1027-1035.

Lind, N. M., Gjedde, A., Moustgaard, A., Olsen, A. K., Jensen, S. B., Jakobsen, S., et al., (2005). Behavioral response to novelty correlates with dopamine receptor availability in striatum of Gottingen minipigs. Behavioural Brain Research, 164, 172-177.

Lucas, L. R., Angulo, J. A., Le Moal, M., McEwen, B. S., y Piazza, P. V. (1998). Neurochemical characterization of individual vulnerability to addictive drugs in rats. Euopeanr Journal of Neuroscience, 10, 3153-3163.

Lusher, J. M., Chandler, C., y Ball, D. (2001). Dopamine D4 receptor gene (DRD4) is associated with Novelty Seeking (NS) and substance abuse: the saga continues. Molecular Psychiatry, 6, 497-499.

Mantsch, J. R., Ho, A., Schlussman, S. D., y Kreek, M. J. (2001). Predictable individual differences in the initiation of cocaine self-administration by rats under extended-access conditions are dose-dependent. Psychopharmacology, 157, 31-39.

Marinelli, M. (2005). The many facets of the locomotor response to a novel environment test: theoretical comment on Mitchell, Cunningham, and Mark (2005). Behavioral Neuroscience, 119, 1144-1151.

Marinelli, M., y White, F. J. (2000). Enhanced vulnerability to cocaine self-administration is associated with elevated impulse activity of midbrain dopamine neurons. Journal of Neuroscience, 20, 8876-8885.

Márquez, C., Nadal, R., y Armario, A. (2004). The hypothalamic-pituitary-adrenal and glucose responses to daily repeated immobilisation stress in rats: individual differences. Neuroscience, 123, 601-612.

Márquez, C., Nadal, R., y Armario, A. (2006). Influence of reactivity to novelty and anxiety on hypothalamicpituitary-adrenal and prolactin responses to two different novel environments in adult male rats. Behavioural Brain Research, 168, 13-22.

Mitchell, J. M., Cunningham, C. L., y Mark, G. P. (2005). Locomotor activity predicts acquisition of selfadministration behavior but not cocaine intake. Behav ioral Neuroscience, 119, 464-472.

Nadal, R., Armario, A., y Janak, P. H. (2002). Positive relationship between activity in a novel environment and operant ethanol self-administration in rats. Psychopharmacology, 162, 333-338.

Nadal, R., Rotllant, D., Marquez, C., y Armario, A. (2005). Perseverance of exploration in novel environments predicts morphine place conditioning in rats. Behavioural Brain Research, 165, 72-79.

Oswald, L. M., Mathena, J. R., y Wand, G. S. (2004). Comparison of hipotalámico-pituitario-adrenal axis hormonal responses to naloxone vs psychologicallyinduced stress. Psychoneuroendocrinology, 29, 371-388.

Oswald, L. M., Wong, D. F., McCaul, M., Zhou, Y., Kuwabara, H., Choi, L., et al., (2005). Relationships among ventral striatal dopamine release, cortisol secretion, and subjective responses to amphetamine. Neuropsychopharmacology, 30, 821-832.

Oswald, L. M., Zandi, P., Nestadt, G., Potash, J. B., Kalaydjian, A. E., y Wand, G. S. (2006). Relationship between cortisol responses to stress and personality. Neuropsychopharmacology, 31, 1583-1591.

Pelloux, Y., Costentin, J., y Duterte-Boucher, D. (2006). Novelty preference predicts place preference conditioning to morphine and its oral consumption in rats. Pharmacology, Biochemistry and Behavior, 84, 43-50.

Piazza, P. V., Deminière, J. M., Le Moal, M., y Simon, H. (1989). Factors that predict individual vulnerability to amphetamine self-administration. Science, 245, 1511-1513.

Piazza, P. V., Deminière, J. M., Maccari, S., Mormède, P., Le Moal, M., y Simon, H. (1990). Individual reactivity to novelty predicts probability of amphetamine selfadministration. Behavioral Pharmacology, 1, 339-345.

Piazza, P.V., Deroche, V., Deminière, J.M., Maccari, S., Le Moal, M., y Simon, H. (1993) Corticosterone in the range of stress-induced levels possesses reinforcing properties: implications for sensation-seeking behaviors. 
Proceedings of the National Academy of Sciences U S A, 90,11738-11742.

Piazza, P. V., Deroche-Gamonent, V., Rougé-Pont, F., y Le Moal, M. (2000). Vertical shifts in self-administration dose-response functions predict a drug-vulnerable phenotype predisposed to addiction. Journal of Neuroscience, 20, 4226-4232.

Piazza, P. V., Maccari, S., Deminière, J. M., Le Moal, M., Mormède, P., y Simon, H. (1991). Corticosterone levels determine individual vulnerability to amphetamine selfadministration. Proceedings of the National Academy of Sciences U S A, 88, 2088-2092.

Pierre, P. J., y Vezina, P. (1997). Predisposition to self-administer amphetamine: the contribution of response to novelty and prior exposure to the drug. Psychopharmacology, 129, 277-284.

Pritchard, L. M., Logue, A. D., Taylor, B. C., Ahlbrand, R., Welge, J. A., Tang, Y., et al., (2006). Relative expression of D3 dopamine receptor and alternative splice variant D3nf mRNA in high and low responders to novelty. Brain Research Bulletin, 70, 296-303.

Ray, J., Hansen, S., y Waters, N. (2006). Links between temperamental dimensions and brain monoamines in the rat. Behavioral Neuroscience, 120, 85-92.

Robinet, P. M., Rowlett, J. K., y Bardo, M. T. (1998). Individual differences in novelty-induced activity and the rewarding effects of novelty and amphetamine in rats. Behavioural Processes, 44, 1-9.

Rougé-Pont, F., Deroche, V., Le Moal, M., y Piazza, P. V. (1998). Individual differences in stress-induced dopamine release in the nucleus accumbens are influenced by corticosterone. European Journal of Neuroscience, 10, 3903-3907.

Rougé-Pont, F., Piazza, P. V., Kharouby, M., Le Moal, M., y Simon, H. (1993). Higher and longer stress-induced increase in dopamine concentrations in the nucleus accumbens of animals predisposed to amphetamine self-administration. A microdialysis study. Brain Research, 602, 169-174.

Savitz, J. B., y Ramesar, R. S. (2004). Genetic variants implicated in personality: a review of the more promising candidates. American Journal of Medical Genet B Neuropsychiatric Genetics, 131, 20-32.

Sax, K. W., y Strakowski, S. M. (1998). Enhanced behavioral response to repeated d-amphetamine and personality traits in humans. Biological Psychiatry, 44, 1192-1195.

Sell, S. L., Dillon, A. M., Cunningham, K. A., y Thomas, M. L. (2005). Estrous cycle influence on individual differences in the response to novelty and cocaine in female rats. Behavioural Brain Research, 161, 69-74.

Sher, K. J., Bartholow, B. D., y Wood, M. D. (2000). Personality and substance use disorders: a prospective study. Journal of Consulting and Clinical Psychology, 68, 818-829.

Shimosato, K., y Watanabe, S. (2003). Concurrent evaluation of locomotor response to novelty and propensity toward cocaine conditioned place preference in mice. Journal of Neuroscience Methods, 128, 103-110.
Sinha, R., Garcia, M., Paliwal, P., Kreek, M. J., y Rounsaville, B. J. (2006). Stress-induced cocaine craving and hypothalamic-pituitary-adrenal responses are predictive of cocaine relapse outcomes. Archives of General Psychiatry, 63, 324-331.

Stansfield, K. H., y Kirstein, C. L. (2005). Neurochemical effects of cocaine in adolescence compared to adulthood. Brain Research Developmental Brain Research, 159, 119-125.

Stead, J. D., Clinton, S., Neal, C., Schneider, J., Jama, A., Miller, S., et al., (2006). Selective breeding for divergence in novelty-seeking traits: heritability and enrichment in spontaneous anxiety-related behaviors. Behavior Genetics, 36, 697-712.

Steimer, T., y Driscoll, P. (2003). Divergent stress responses and coping styles in psychogenetically selected Roman high-(RHA) and low-(RLA) avoidance rats: behavioural, neuroendocrine and developmental aspects. Stress, 6 , 87-100.

Stoops, W. W., Lile, J. A., Robbins, C. G., Martin, C. A., Rush, C. R., y Kelly, T. H. (2007). The reinforcing, subject-rated, performance, and cardiovascular effects of d-amphetamine: influence of sensation-seeking status. Addictive Behavior, 32, 1177-1188.

Suto, N., Austin, J. D., y Vezina, P. (2001). Locomotor response to novelty predicts a rat's propensity to self-administer nicotine. Psychopharmacology, 158, 175-180.

Tyrka, A. R., Wier, L. M., Anderson, G. M., Wilkinson, C. W., Price, L. H., y Carpenter, L. L. (2007). Temperament and response to the Trier Social Stress Test. Acta Psychiatrica Scandinavica, 115, 395-402.

Wand, G. S., Oswald, L. M., McCaul, M. E., Wong, D. F., Johnson, E., Zhou, Y., et al., (2007). Association of Amphetamine-Induced Striatal Dopamine Release and Cortisol Responses to Psychological Stress. Neuropsychopharmacology, en prensa.

White, T. L., Grover, V. K., y de Wit, H. (2006a). Cortisol effects of $\mathrm{D}$-amphetamine relate to traits of fearlessness and aggression but not anxiety in healthy humans. Pharmacology, Biochemistry and Behavior, 85, 123-131.

White, T. L., Lott, D. C., y de Wit, H. (2006b). Personality and the subjective effects of acute amphetamine in healthy volunteers. Neuropsychopharmacology, 31, 1064-1074.

Wills, T. A., Vaccaro, D., y McNamara, G. (1994). Novelty seeking, risk taking, and related constructs as predictors of adolescent substance use: an application of Cloninger's theory. Journal of Substance Abuse, 6, 1-20.

Wooters, T. E., Dwoskin, L. P., y Bardo, M. T. (2006). Age and sex differences in the locomotor effect of repeated methylphenidate in rats classified as high or low novelty responders. Psychopharmacology, 188, 18-27.

Wrona, D., Jurkowski, M., Luszawska, D., Tokarski, J., y Trojniar, W. (2003). The effects of lateral hypothalamic lesions on peripheral blood natural killer cell cytotoxicity in rats hyper- and hyporesponsive to novelty. Brain, Behavior and Immunity, 17, 453-461. 
Wrona, D., Jurkowski, M. K., y Tokarski, J. (2004). Blood and spleen natural killer cell cytotoxicity after exposure to open field stress in rats: the effect of spontaneous locomotor activity. Journal of Neuroimmunology, 150, 88-97.

Wrona, D., Sukiennik, L., Jurkowski, M. K., Jurkowlaniec, E., Glac, W., y Tokarski, J. (2005). Effects of amphetamine on NK-related cytotoxicity in rats differing in locomotor reactivity and social position. Brain, Behavior and Immunity, 19, 69-77.
Zheng, X., Ke, X., Tan, B., Luo, X., Xu, W., Yang, X., et al., (2003). Susceptibility to morphine place conditioning: relationship with stress-induced locomotion and novelty-seeking behavior in juvenile and adult rats. Pharmacology, Biochemistry and Behavior, 75, 929-935.

Zuckerman, M. (1986). Sensation seeking and the endogenous deficit theory of drug abuse. NIDA Research Monograph, 74, 59-70. 